\title{
O modelo Muskingum-Cunge-Todini em rios com planície de inundação
}

\section{The Muskingum-Cunge-Todini streamflow routing model in floodplain rivers}

\author{
Paulo Rógenes Monteiro Pontes ${ }^{1}$ e Walter Collischonn ${ }^{2}$
}

\begin{abstract}
${ }^{1}$ Doutorando do Instituto de Pesquisas Hidráulicas-Universidade Federal do Rio Grande do Sul - Porto Alegre - RS - Brasil
\end{abstract}

$$
\text { p.rogenes@gmail.com }
$$

${ }^{2}$ Professor Dr. noInstituto de Pesquisas Hidráulicas-Universidade Federal do Rio Grande do Sul - Porto Alegre - RS - Brasil collischonn@iph.ufrgs.br

\section{RESUMO}

Esse artigo é o segundo artigo de uma série que analisa o modelo Muskingum-Cunge-Todini (MCT). No primeiro artigo (Pontes e Collischonn, 2012) é feita uma comparação entre os modelos simplificados de propagação de vazão MCT, MCL (Muskingum-Cunge linear) e MCNL (Muskingum-Cunge não linear). Além disso, também são realizados testes em um sistema real.No atual artigo é apresentada uma modificação do modelo MCT para considerar o fluxo em rios com planície de inundação, onde a velocidade na área alagada é significativamente menor do que a velocidade da água na calha principal, o que afeta fortemente a celeridade da onda de cheia. Também foram realizados testes de conservação de volume variando declividade, rugosidade, discretização temporal e espacial. Além disso, foi realizada uma análise acerca do valor de $\Delta x$ ideal, para evitar problemas de volume ou instabilidade numérica, e acerca dos critérios de aplicabilidade descritos por Ponce (1989). O modelo MCT considerando planície de inundação foi comparado com o modelo bidrodinâmico HEC-RAS já consagrado na literatura, e um modelo Muskingum-Cunge não linear (Tucci, 2005). Os resultados mostram que o modelo MCT modificado para considerar a planície de inundação conserva o volume nos casos de variação de declividade e rugosidade. Com relação aos valores de $\Delta x$, 0 modelo apresenta certa sensibilidade. Valores muito altos de $\Delta x$ podem originar erros de vazão de pico e tempo de ocorrência da vazão de pico, além de instabilidade nos bidrogramas. Os mesmos problemas podem ser identificados no modelo HEC-RAS quando adotado $\Delta x$ altos. Também foi sugerida uma formulação para o cálculo do $\Delta x$ ideal. Esse novo valor diminui a ocorrência de erros de volume e instabilidade nos hidrogramas.

Palavras Chave: Conservação de volume. Muskingum-Cunge não linear. Planície de Inundação

\section{ABSTRACT:}

This paper is the second of two papers in a series which analyzes and improves the Muskingum-Cunge-Todini (MCT) model. In the first paper the simplified stream flow model and HEC-RAS are compared. The volume error was analyzed. The current paper presents a modification in the MCT model to account for the flow in floodplain rivers, whose velocity in flooded areas is significantly lower than the velocity in the main channel. This paper also shows the volume conservation tests and analysis of DX to avoid volume errors and numerical stability problems. The results show that the improved MCT model is conservative regarding volume.

Keywords: Volume conservation. Non-linear Muskingum-Cunge. Floodplain 


\section{INTRODUÇÃO}

Os cálculos da propagação de ondas de cheias em rios podem ser realizados atualmente de forma relativamente simples com o uso de modelos hidrodinâmicos que resolvem numericamente as equações de Saint-Venant.

Em geral, os modelos hidrodinâmicos representam muito bem os efeitos de amortecimento, translação, efeitos de jusante e a planície de inundação, além de propagarem a onda de cheia conservando o volume dos hidrogramas e representando bem as vazões de pico. Em contrapartida, os modelos hidrodinâmicos podem exigir um alto custo computacional, especialmente se o modelo necessita ser aplicado um grande número de vezes, ou se a simulação é realizada para vários períodos de tempo. Diante disso, modelos mais simplificados de propagação de vazão ainda têm uma grande importância, uma vez que eles exigem menos dados de entrada e, normalmente, são mais eficientes computacionalmente.Esses modelos visam cada vez mais contemplar os efeitos representados por modelos hidrodinâmicos.

Um dos modelos simplificados mais importantes de escoamento em rios continua sendo o modelo de Muskingum, proposto por McCarthy em 1939 (TUCCI, 2005). Esse modelo foi modificado por Cunge, em 1969, para que os seus parâmetros $\mathrm{K}$ e X variassem no tempo, de acordo com a vazão a ser propagada. Essa versão passou a ser conhecida como modelo Muskingum-Cunge não linear (MCNL). A versão em que os valores de $\mathrm{K}$ e $\mathrm{X}$ não se alteram ao longo do tempo é conhecida como Muskingum-Cunge linear (MCL) (PONCE, 1989; TUCCI, 2005).

O método MCNL apresenta grandes vantagens quando a celeridade da onda de cheia varia com a vazão. Isto é especialmente importante em rios com planícies de inundação, em que a celeridade da onda de cheia é bastante diferente nas condições de escoamento contido na calha, ou escoamento que inunda a planície. Nestas condições, o modelo MCL não representa tão bem as vazões de pico como o modelo MCNL (PERUMAL et al., 2001). Tucci (2005) e Silva et al. (2003), por exemplo, mostram algumas vantagens da utilização do método MCNL no rio Jacuí - RS em escoamentos com planície de inundação. Entretanto, diversos autores demonstraram que o método MCNL pode ter um erro de conservação de massa da ordem de $8 \%$ a 10\% (TANG; SAMUELS, 1999; FAILACHE et al., 2004).

Ao longo dos últimos 20 anos o problema da conservação de volume no modelo MCNL foi analisado por diversos autores, que procuraram resolver a questão de diversas formas (PERUMAL, 1994a,b; CAPPELAERE, 1997; TODINI, 2007; PRICE, 2009).

Uma das propostas mais simples, e ao mesmo tempo mais promissoras, foi desenvolvida por Todini (2007). Este autor percebeu uma inconsistência na forma como eram obtidas as equações para aplicação do modelo MCNL a partir das equações originais de Muskingum-Cunge e desenvolveu uma metodologia que contempla a conservação de volume, denominada método Muskingum-Cunge-Todini (MCT).

Pontes e Collischonn (2012) compararam os modelos MCT, MCL e duas versões do modelo MCNL em um canal hipotético com seção transversal retangular e mostraram que o modelo MCT apresentou melhor desempenho em termos de conservação de volume. Os autores mostraram que o modelo pode representar casos reais satisfatoriamente.

Apesar dos testes realizados em seções retangulares surgiu a dúvida acerca da capacidade dos modelos simplificados de propagação de vazão conservar volume em rios com planície de inundação da mesma forma que modelos hidrodinâmicos o fazem.

Existem diversas alternativas para representar tanto o fluxo no canal principal como na planície de inundação. Com relação à planície de inundação, pode-se usar metodologia de seções compostas acoplando isso a modelos hidrodinâmicos ou modelos simplificados de propagação de vazão. Outra forma consiste no uso de modelos bidimensionais ou tridimensionais para representar o escoamento na planície. Além disso, pode-se usar um modelo de células acoplado a um modelo unidimensional para representação do fluxo na planície do canal.

Alguns trabalhos desenvolvidos nessa última década abordam a combinação de modelos diferentes para planície e para o canal principal (PAZ, 2010; HORRITTE BATES, 2001; PAIVA, 2009).

O presente trabalho, o segundo de uma série de dois artigos sobre propagação de vazão utilizando o modelo MCT (PONTES; COLLISCHONN, 2012), apresenta uma extensão do modelo MCT para considerar o fluxo em rios com planície de inundação, onde a velocidade é significativamente menor do que a velocidade da água na calha principal, o que afeta fortemente a celeridade da onda de cheia. Também são realizados testes de conservação de volume para uma ampla faixa de condições, e alterando características do rio e opções de discretização espacial e temporal. Esse modelo é comparado com o modelo hidrodinâmico HEC-RAS (USACE, 2008) já consagrado na literatura, e um modelo Muskingum-Cunge não linear (TUCCI, 2005).

\section{MODELOS HIDRODINÂMICOS}

Os modelos hidrodinâmicos unidimensionais utilizam as equações da conservação de massa e da quantidade de movimento para representar o escoamento em rios e canais. Essas duas equações combinadas formam o sistema de equações conhecido como equações de Saint-Venant:

$$
\begin{aligned}
& \frac{\partial A}{\partial t}+\frac{\partial Q}{\partial x}=0, \\
& \frac{\partial Q}{\partial t}+\frac{\partial\left(Q^{2} / A\right)}{\partial x}+g A \frac{\partial h}{\partial x}=g A S_{0}-g A S_{f},
\end{aligned}
$$

onde

$\mathrm{A}\left[\mathrm{L}^{2}\right]$ é a área da seção transversal, $\mathrm{Q}\left[\mathrm{L}^{3} / \mathrm{T}\right]$ é a vazão, q $\left[\mathrm{L}^{2} / \mathrm{T}\right]$ é a vazão de contribuição lateral, t [T] e x [L] são respectivamente o tempo e a distância longitudinal, $g\left[\mathrm{~L} / \mathrm{T}^{2}\right]$ é a aceleração da gravidade, $[\mathrm{L} / \mathrm{L}]$ é a declividade do fundo do rio, $[\mathrm{L} / \mathrm{L}]$ é a declividade da linha de energia e $\mathrm{h}[\mathrm{L}]$ a profundidade da linha d'água, que pode ser dada pela equação a seguir.

$$
S_{f}=\frac{Q^{2} \cdot n^{2}}{A^{2} \cdot R^{4 / 3}},
$$


onde $\mathrm{n}$ é a rugosidade $\left[\mathrm{L}^{-1 / 3} / \mathrm{T}\right]$ e $\mathrm{R}$ é o raio hidráulico.

É importante citar que, como o conjunto de equações na forma completa é formado por um sistema de equações diferenciais de primeira ordem, do tipo hiperbólico, não existe uma solução analítica. Sendo assim, é necessária a utilização de técnicas numéricas para a resolução das equações.

A técnica numérica mais freqüentemente utilizada é o método das diferenças finitas. Este método aproxima as derivadas parciais analíticas para derivadas numéricas, e pode ser usado em esquemas explícitos ou implícitos.

Quando a estimativa da variável no tempo i+1 é feita a partir de resultados da mesma variável no tempo i, o esquema é chamado explícito. Nos esquemas implícitos, o cálculo da variável no tempo i+1 não pode ser explicitado, formando assim um sistema de equações.

Vários esquemas numéricos podem ser utilizados para a resolução dessas equações. Um dos mais importantes é o esquema implícito de quatro pontos de Preissmann (Cunge et al.,1980), utilizado, por exemplo, no modelo HEC-RAS (USACE, 2008). Outro método para a resolução das equações de Saint-Venant é apresentado por Silva et al. (2003). É chamado de Método Explícito Difusivo de Diferenças Finitas.

A partir do desenvolvimento desses métodos de resolução de equações diferenciais, uma variedade de programas foi desenvolvida utilizando os modelos hidrodinâmicos em sua forma completa, como é o caso dos programas HEC-RAS (USACE, 2008) e MIKE11 (THOMPSON et al., 2004; ZHANG et al., 2008).

Apesar da facilidade na resolução das equações completas de Saint-Venant, graças a essas técnicas de resolução e ao desenvolvimento desses programas, é importante citar que estudos envolvendo modelos hidrodinâmicos requerem atenção em alguns aspectos. Um exemplo disso é a questão da melhor representatividade do escoamento nas planícies de inundação. Pesquisas vêm sendo desenvolvidas com o intuito de melhorar a predição de áreas alagadas e entender melhor o escoamento nessas planícies (PAZ, 2006).

\section{MODELOS SIMPLIFICADOS MCL E MCNL}

Em 1969, Cunge concluiu que o método de Muskingum é equivalente a uma solução numérica da equação hiperbólica da onda cinemática (Equação 9). Este autor também concluiu que a atenuação da onda de cheia que acontece na propagação utilizando a solução numérica da equação de onda cinemática é devido à difusão numérica em função do esquema numérico de diferenças finitas adotado para representar as derivadas da equação 4 .

$$
\frac{\partial \mathrm{Q}}{\partial \mathrm{t}}+\mathrm{c} \frac{\partial \mathrm{Q}}{\partial \mathrm{x}}=0
$$

Cunge utilizou um esquema numérico de quatro pontos, como ilustrado na figura 1, e aproximou as derivadas através das equações 5 e 6 .

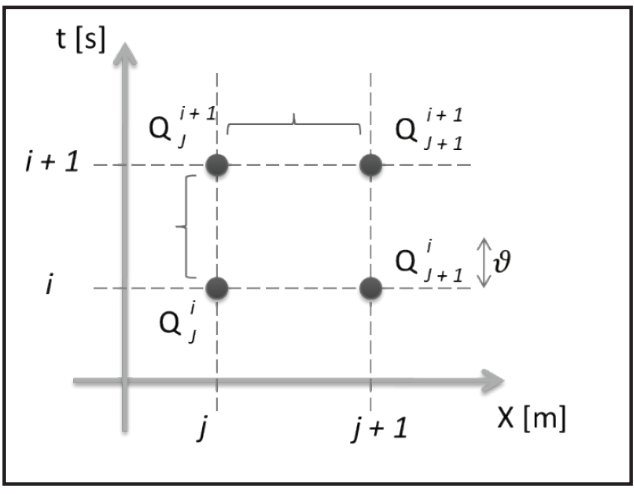

Figura 1 - Esquema numérico de quatro pontos utilizado no método Muskingum-Cunge. Os índices i e j representam tempo e espaço respectivamente. $\mathrm{Q}$ é a vazão, t o eixo tempo e x o eixo espaço

As derivadas são aproximadas da seguinte forma:

$$
\begin{aligned}
& \frac{\partial Q}{\partial t}=\frac{\varepsilon\left(Q_{j}^{i+1}-Q_{j}^{i}\right)+(1-\varepsilon)\left(Q_{j+1}^{i+1}-Q_{j+1}^{i}\right)}{\Delta t}, \\
& \frac{\partial Q}{\partial x}=\frac{\vartheta\left(Q_{j+1}^{i+1}-Q_{j}^{i+1}\right)+(1-\vartheta)\left(Q_{j+1}^{i}-Q_{j}^{i}\right)}{\Delta x} .
\end{aligned}
$$

Os índices i e j representam tempo e espaço respectivamente. Q é a vazão e os termos $\varepsilon$ e $\vartheta$ são parâmetros ponderadores.

Substituindo essas equações 5 e 6 na equação 4, obtém-se:

$$
\frac{\varepsilon\left(Q_{j}^{i+1}-Q_{j}^{i}\right)+(1-\varepsilon)\left(Q_{j+1}^{i+1}-Q_{j+1}^{i}\right)}{\Delta t}+\frac{c}{2} \frac{\left(Q_{j+1}^{i+1}-Q_{j}^{i+1}\right)+\left(Q_{j+1}^{i}-Q_{j}^{i}\right)}{\Delta x}=0,
$$

(Considerando $\vartheta=0.5$, ou seja, o esquema numérico centrado). Após algumas manipulações, pode-se obter a seguinte equação:

$$
Q_{j+1}^{i+1}=C 1 Q_{j}^{i+1}+C 2 Q_{j}^{i}+C 3 Q_{j+1}^{i}
$$

onde:

$$
\begin{aligned}
& \mathrm{C} 1=\frac{-2 \Delta \mathrm{x} \varepsilon+\mathrm{c} \Delta \mathrm{t}}{2 \Delta \mathrm{x}(1-\varepsilon)+\mathrm{c} \Delta \mathrm{t}}, \\
& \mathrm{C} 2=\frac{2 \Delta \mathrm{x} \varepsilon+\mathrm{c} \Delta \mathrm{t}}{2 \Delta \mathrm{x}(1-\varepsilon)+\mathrm{c} \Delta \mathrm{t}}, \\
& \mathrm{C} 3=\frac{2 \Delta \mathrm{x} \varepsilon-\mathrm{c} \Delta \mathrm{t}}{2 \Delta \mathrm{x}(1-\varepsilon)+\mathrm{c} \Delta \mathrm{t}},
\end{aligned}
$$

Cunge (1969) sugeriu uma equação para o parâmetro $\varepsilon$, onde a difusão numérica seria equivalente à difusão real:

$$
\varepsilon=0.5\left(1-\frac{\mathrm{Q}}{\mathrm{c} \Delta \mathrm{xBS0}}\right)
$$

onde B e S0 correspondem a largura do rio [L] e a declividade de fundo $[\mathrm{L} / \mathrm{L}]$, respectivamente. 
Considerando o valor do parâmetro sugerido por Cunge, pode-se reescrever as equações 9 a 11 na forma apresentada a seguir:

$$
\begin{aligned}
& \mathrm{C} 1=\frac{-1+\mathrm{C}+\mathrm{D}}{1+\mathrm{C}+\mathrm{D}}, \\
& \mathrm{C} 2=\frac{1+\mathrm{C}-\mathrm{D}}{1+\mathrm{C}+\mathrm{D}}, \\
& \mathrm{C} 3=\frac{1-\mathrm{C}+\mathrm{D}}{1+\mathrm{C}+\mathrm{D}},
\end{aligned}
$$

onde C é o número de Courant e D é o coeficiente de difusão, dado por:

$$
\begin{aligned}
& \mathrm{C}=\frac{\mathrm{c} \Delta \mathrm{t}}{\Delta \mathrm{x}}, \\
& \mathrm{D}=\frac{\mathrm{Q}}{\mathrm{c} \Delta \mathrm{xBS} 0} .
\end{aligned}
$$

O parâmetro $\varepsilon$ é estimado com base em uma vazão de referência. Após a quantificação dessa vazão de referência, calcula-se a celeridade, e os outros parâmetros necessários para a obtenção do hidrograma de saída.

No modelo Muskingum-Cunge Linear (MCL), essa vazão de referência é fixa para todo o período de cálculo. Tucci (2005) sugere que a vazão de referência seja aproximadamente $70 \%$ da vazão máxima do hidrograma de entrada no trecho.

No modelo Muskingum-Cunge Não Linear (MCNL), a vazão de referência é calculada em cada passo de tempo de simulação. Desta forma, $\varepsilon$ também varia em cada passo de tempo.

Diferentes formas de estimar a vazão de referência foram propostas (FAILLACHE et al., 2004; TANGE; SAMUELS, 1999; PONCE, 1989).

Dois esquemas para a estimação da vazão de referência são mais usuais: (a) o esquema de três pontos explícito - chamado aqui de MCNL3 - (equação 18); e (b) o esquema de 4 pontos implícito - MCNL4 - (equação 19)

$$
\begin{aligned}
& \operatorname{Qref}(i, j)=\frac{Q_{j}^{i}+Q_{j}^{i+1}+Q_{j+1}^{i}}{3} \\
& \operatorname{Onde~} c(i, j)=c(Q \operatorname{ref}(i, j)) \\
& \operatorname{Qref}(i, j)=\frac{Q_{j+1}^{i+1}+Q_{j}^{i}+Q_{j}^{i+1}+Q_{j+1}^{i}}{4}, \\
& \text { Onde } c(i, j)=c(Q \operatorname{ref}(i, j))
\end{aligned}
$$

O método MCNL4 exige um processo iterativo para solução da equação, em função da presença do termo $Q^{i+1}$ e por ser um método implícito. O método MCNL3 é explícito, logo não precisa das iterações para encontrar o termo $\mathrm{Q}^{i+1}$. $\mathrm{O}$ termo c $(i, j)$ é a celeridade $[\mathrm{L} / \mathrm{T}]$ no tempo i e espaço ou trecho $j$.

Operacionalmente, o modelo MCNL é aplicado utilizando uma tabela que relaciona a vazão no sub-trecho com a celeridade e os parâmetros do modelo. Para cada passo de tempo uma vazão de referência é estimada a partir da equação 18 ou da equação 19, e os valores dos parâmetros são definidos de acordo com a relação tabular.

\section{MODELO MUSKINGUM-CUNGE-TODINI (MCT)}

Todini (2007) detectou dois problemas no método MCNL. Um dos problemas está relacionado com a conservação de massa ou volume.

No modelo Muskingum-Cunge não linear os parâmetros $\mathrm{k}$ e $\varepsilon$ devem variar em cada passo de tempo e em cada trecho. Operacionalmente, essa premissa é obedecida, porém Todini percebeu que a variação de $\mathrm{k}$ e $\varepsilon$ no tempo não acontece na concepção matemática dos modelos. Conceitualmente, o modelo não linear utiliza o equacionamento inicial do modelo linear, gerando problemas de conservação de volume.

A resolução desse problema consiste em uma nova derivação das equações do modelo Muskingum-Cunge assumindo a variação dos parâmetros $\mathrm{k}$ e $\varepsilon$ no tempo como mostra a equação 20 .

$$
\frac{\mathrm{d}(\mathrm{k} \varepsilon \mathrm{I})}{\mathrm{dt}}+\frac{\mathrm{d}(\mathrm{k}(1-\varepsilon) \mathrm{Q})}{\mathrm{dt}}=\mathrm{I}-\mathrm{Q}
$$

A variável I corresponde às vazões de entrada no sistema. As derivadas na equação 20 são aproximadas por derivadas numéricas, resultando na equação 21 .

$$
\begin{aligned}
& \frac{(k \varepsilon)_{i+1} I_{i+1}-(k \varepsilon)_{i} I_{i}}{\Delta t}+\frac{(k(1-\varepsilon))_{i+1} Q_{i+1}-(k(1-\varepsilon))_{i} Q_{i}}{\Delta t}=\frac{I_{i+1}+I_{i}}{2}- \\
& \frac{Q_{i+1}+Q_{i}}{2} .
\end{aligned}
$$

Após algumas operações matemáticas, pode-se reescrever as equações 13,14 e 15 com os parâmetros $\mathrm{k}$ e $\varepsilon$ variando no tempo:

$$
\begin{aligned}
& \mathrm{C} 1=\frac{-1+\mathrm{C}_{i+1}+\mathrm{D}_{\mathrm{i}+1}}{1+\mathrm{C}_{\mathrm{i}+1}+\mathrm{D}_{\mathrm{i}+1}}, \\
& \mathrm{C} 2=\frac{\mathrm{C}_{\mathrm{i}+1}}{\mathrm{C}_{\mathrm{i}}} \frac{1+\mathrm{C}_{\mathrm{i}}-\mathrm{D}_{\mathrm{i}}}{1+\mathrm{C}_{\mathrm{i}+1}+\mathrm{D}_{\mathrm{i}+1}}, \\
& \mathrm{C} 3=\frac{\mathrm{C}_{\mathrm{i}+1}}{\mathrm{C}_{\mathrm{i}}} \frac{1-\mathrm{C}_{\mathrm{i}}+\mathrm{D}_{\mathrm{i}}}{1+\mathrm{C}_{\mathrm{i}+1}+\mathrm{D}_{\mathrm{i}+1}},
\end{aligned}
$$

Essa modificação permite a conservação de volume dos hidrogramas propagados.

O segundo problema do método MCNL está associado às equações de armazenamento. A derivação dessas equações permite calcular o armazenamento no tempo $\mathrm{t}+1$ de duas formas diferentes:

$$
\begin{aligned}
& \frac{S_{i+1}-S_{i}}{\Delta t}=\frac{I_{i+1}+I_{i}}{2}-\frac{Q_{i+1}+Q_{i}}{2}, \\
& S_{i+1}=k \varepsilon I_{i+1}+k(1-\varepsilon) Q_{i+1} .
\end{aligned}
$$

Os resultados obtidos pelas equações 25 e 26 apresentam valores de armazenamento S(i+1) diferentes e que não estão de acordo com as condições de regime permanente. Todini (2007) mostra que a equação 25 não respeita as condições iniciais de armazenamento em regime permanente e o armazenamento calculado pela equação 26 apresenta valores menores do que 
as condições de regime permanente.

Em regime permanente as vazões de entrada e saída em um determinado trecho devem ser iguais, sem considerar o amortecimento da onda, mas apenas uma translação "k". O armazenamento é calculado pela equação 27

$$
\mathrm{S}_{\mathrm{i}+1}=\mathrm{kI}_{\mathrm{i}+1}=\mathrm{kQ}_{\mathrm{i}+1}
$$

O armazenamento também pode ser calculado pela equação 26 baseado no equacionamento inicial do modelo Muskingum (KOUSSIS, 2009).

$$
\mathrm{S}=\mathrm{A} \Delta \mathrm{x}=\frac{\mathrm{Q}}{\mathrm{v}} \Delta \mathrm{x}=\mathrm{k} \mathrm{Q},
$$

onde A é a área da seção $\left[\mathrm{L}^{2}\right] ; \Delta \mathrm{x}$ é o trecho ou comprimento [L]; Q é a vazão $\left[\mathrm{L}^{3} / \mathrm{T}\right]$, v é a velocidade média na seção $[\mathrm{L} / \mathrm{T}]$ e k é referente à translação da vazão [T].

Em contrapartida, o modelo derivado por Cunge em 1969 (Todini, 2007; Ponce, 1989) considera que a onda translada com uma celeridade "c" e translação "k", dado por:

$$
\mathrm{k}=\frac{\Delta \mathrm{x}}{\mathrm{c}}
$$

Para resolver a inconsistência com o regime permanente deve-se partir da premissa que o modelo Muskingum (não linear) respeita essas condições. Todini, então, introduziu um parâmetro $\beta$, que corresponde à razão entre celeridade e velocidade da onda.

Considerando esse novo parâmetro, pode-se reescrever as equações 16 e 17 na forma apresentada a seguir:

$$
\begin{aligned}
& \mathrm{C}^{*}=\frac{\mathrm{c} \Delta \mathrm{t}}{\Delta \mathrm{x}} \frac{1}{\beta}, \\
& \mathrm{D}^{*}=\frac{\mathrm{Q}}{\mathrm{c} \Delta \mathrm{xBS} 0} \frac{1}{\beta},
\end{aligned}
$$

Portanto, deve-se reescrever C1, C2 e C3 em função de $\mathrm{C}^{*}$ e $\mathrm{D}^{*}$ para que o modelo MCT conserve volume e respeite a condição de armazenamento em regime permanente.

\section{Algoritmo do método MCT}

Nesta seção descreve-se um resumo do algoritmo computacional referente ao método MCT para nortear a implementação e otimizar os custos computacionais. É importante citar que as equações a seguir são válidas para um canal com seção retangular.

Nesse tópico o intervalo temporal foi representado pelo índice "i” e o intervalo espacial é representado por “j”.

O primeiro passo do algoritmo é estimar a vazão no tempo i+1 do hidrograma de saída. Inicialmente o valor é:

$$
\mathrm{Q} 0=Q_{j+1}^{\mathrm{i}}+\mathrm{Q}_{\mathrm{j}}^{\mathrm{i}+1}-\mathrm{Q}_{\mathrm{j}}^{\mathrm{i}}
$$

Com essa vazão, calculam-se as vazões de referência nos intervalos de tempo i e i+1:

$$
\begin{gathered}
\operatorname{Qref}_{j+1}^{i}=\frac{Q_{j}^{i}+Q_{j+1}^{i}}{2}, \\
\operatorname{Qref}_{j+1}^{i+1}=\frac{Q_{j}^{i+1}+Q 0}{2} .
\end{gathered}
$$

Com base nessas vazões de referência, calcula-se os níveis (y) de água na seção de jusante nos intervalos de tempo i e i+1:

$$
\begin{aligned}
& y_{j+1}^{i}=\alpha \operatorname{Qref}_{j+1}^{i}, \\
& y_{j+1}^{i+1}=\alpha Q \operatorname{Qref}_{j+1}^{i+1}, \\
& \alpha=\frac{n^{0.6}}{s 0^{0.3} B^{0.6}},
\end{aligned}
$$

onde y corresponde ao nível de água [L] e o termo alfa é parte da equação de Manning reorganizada.

Usando ainda a vazão de referência, calculam-se as celeridades em cada intervalo de tempo:

$$
\begin{aligned}
& c_{j+1}^{i}=\frac{5}{3} \frac{s 0^{0.3}}{n^{0.6} B^{0.4}} \operatorname{Qref}_{j+1}^{i}, \\
& c_{j+1}^{i+1}=\frac{5}{3} \frac{S 0^{0.3}}{n^{0.6} B^{0.4}} \operatorname{Qref}_{j+1}^{i+1},
\end{aligned}
$$

Aqui, assumimos que o raio hidráulico é equivalente ao nível da água (i.e. canais que apresentam largura muito superior a profundidade). A variável n é a rugosidade e a variável B a largura do rio.

Com base na vazão de referência, na área de seção transversal e na celeridade pode-se calcular o fator de correção $\beta^{*}$ :

$$
\begin{aligned}
& \beta_{j+1}^{* i}=\frac{c_{j+1}^{i} A_{j+1}^{i}}{\operatorname{Qref} f_{j+1}^{i}}, \\
& \beta_{j+1}^{* i+1}=\frac{c_{j+1}^{i+1} A_{j+1}^{i+1}}{\operatorname{Qref} f_{j+1}^{i+1}},
\end{aligned}
$$

o número de Courant $\left(\mathrm{C}^{*}\right)$ :

$$
\begin{aligned}
& C^{* i}{ }_{j+1}^{i}=\frac{c_{j+1}^{i} \Delta t}{\beta *_{j+1}^{i} \Delta x}, \\
& C^{* i+1}=\frac{c_{j+1}^{i+1} \Delta t}{\beta *_{j+1}^{i+1} \Delta x},
\end{aligned}
$$

e o coeficiente de Difusão (D*):

$$
\begin{aligned}
& D_{j+1}^{* i}=\frac{\operatorname{Qref}_{j+1}^{i}}{c_{j+1}^{i} \beta *_{j+1}^{i} \Delta \times B S 0}, \\
& D_{j+1}^{* i+1}=\frac{Q r f_{j+1}^{i+1}}{c_{j+1}^{i+1} \beta *_{j+1}^{i+1} \Delta x B S 0} .
\end{aligned}
$$

Na seqüência, calcula-se os parâmetros C1, C2 e C3 do método proposto por Todini (2007): 


$$
\begin{aligned}
& \mathrm{C} 1=\frac{-1+\mathrm{C}_{\mathrm{j}+1}^{* i+1}+\mathrm{D}_{\mathrm{j}+1}^{* i+1}}{1+\mathrm{C}_{\mathrm{j}+1}^{*+1}+\mathrm{D}_{\mathrm{j}+1}^{* i+1}}, \\
& C 2=\frac{1+C_{j+1}^{* i}-D_{j+1}^{* i}}{1+C_{j+1}^{*}{ }_{j}^{i+1}+D^{*}{ }_{j+1}^{i+1}} \text {, } \\
& \mathrm{C} 3=\frac{1-\mathrm{C}_{\mathrm{j}+1}^{* i}+\mathrm{D}_{\mathrm{j}+1}^{* i}}{1+\mathrm{C}_{\mathrm{j}+1}^{* i+1}+\mathrm{D}_{\mathrm{j}+1}^{*+1}} \text {. }
\end{aligned}
$$

O último passo é calcular a vazão de saída no tempo i+1 pela fórmula tradicional:

$$
Q_{j+1}^{i+1}=C 1 Q_{j}^{i+1}+C 2 Q_{j}^{i}+C 3 Q_{j+1}^{i} \text {. }
$$

É importante que esta seqüência de cálculo se repita pelo menos duas vezes, para que se possa eliminar a influência da estimativa inicial para $Q_{j+1}^{i+1}$.

\section{REPRESENTAÇÃO DA PLANÍCIE DE INUNDAÇÃO}

O comportamento da onda de cheia é afetado consideravelmente pela interação entre canal e planície. Por isso, em rios onde existe planície de inundação deve-se levar em consideração a representação da mesma no modelo utilizado.

O armazenamento na planície de inundação é um dos mais importantes aspectos que contribuem para o amortecimento dos hidrogramas de cheia. Segundo Price (2009), modelos que consideram esse efeito são muito mais precisos quando relacionados aos que não consideram, além de representarem o sistema simulado de maneira mais aproximada quando comparados com modelos hidrodinâmicos. As trocas de água entre calha e planície influenciam diretamente na celeridade da onda como pode ser visto na Figura 2. Nota-se que enquanto a água permanece na calha $(\mathrm{Q}=\mathrm{Q} 1)$, a celeridade tende a crescer. Isso acontece devido às relações geométricas da seção. Assim que a água passa para a planície de inundação $(\mathrm{Q}=\mathrm{Q} 2)$, a celeridade decresce até um valor muito baixo e rapidamente. A partir do momento que a água está estabilizada na seção $(\mathrm{Q}=\mathrm{Q} 3)$ não há um aumento significativo na largura da seção transversal, fazendo com que a celeridade aumente, mas de uma forma mais branda.

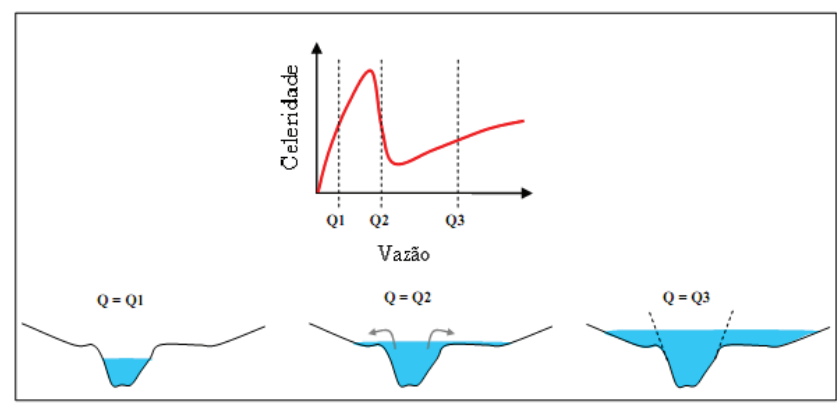

Figura 2 - Efeito do armazenamento na planície de inundação na celeridade

Fonte: Paiva, 2009

\section{Critérios de Aplicabilidade e $\Delta x$ ideal dos modelos simplificados}

Primeiramente deve-se saber que existe uma diferença entre "aplicabilidade" e "estabilidade". O último termo está associado com o esquema numérico. Para saber a estabilidade de um esquema numérico pode ser utilizado o critério de Newmann.

Porém analisar estabilidade de esquema numérico não é o foco deste trabalho. O esquema numérico mais utilizado nos modelos de propagação de vazão é o de Preissmann, no qual é sabido que o esquema é estável para (parâmetro ponderador do esquema numérico). Todo o equacionamento para chegar a esse valor não será mostrado aqui, mas é de fácil acesso na literatura.

Com relação à aplicabilidade dos modelos, essa sim será tratada aqui, é importante saber o porquê da sua importância. Modelos simplificados de propagação, como já dito em outras partes desse trabalho, são limitados por simplificações nos termos da equação dinâmica (ou da quantidade de movimento). Essas limitações não permitem que tais modelos sejam utilizados para qualquer situação. Devido a isso, alguns critérios de aplicabilidade foram definidos por alguns estudiosos da área.

Como exemplo pode-se citar as inequações propostas por Ponce (1989) para onda cinemática e modelo tipo difusão respectivamente:

$$
\frac{t . S 0 . V}{h} \geq 85
$$

onde: t é o tempo de subida do hidrograma de entrada, S0 é a declividade, v é a velocidade média na seção transversal e h a profundidade média na seção transversal.

$$
\frac{\mathrm{t}^{\mathrm{S} 0 . \mathrm{g}^{0.5}}}{\mathrm{~h}^{0.5}} \geq 15
$$

o parâmetro g é a aceleração da gravidade.

Obs.: Todos os valores nas unidades do sistema internacional SI.

Deve-se ter um cuidado especial na hora de afirmar a escolha de um tipo de modelo baseado nos resultados desses testes. Perumal et al.(2007) mostram que os critérios supracitados podem apresentar uma deficiência em alguns casos.

Outra equação que pode ser citada é a apresentada por Fread (1993) com base em um trabalho de Jones (1981) que define a discretização espacial ideal para os modelos de Muskingum-Cunge. A equação é a seguinte:

$$
\Delta x \cong 0,5 \cdot c_{0} \cdot \Delta t\left[1+\left(1+1,5 \cdot \frac{Q_{0}}{B \cdot \Delta t \cdot S_{0} \cdot c_{0}^{2}}\right)^{2}\right] \text {. }
$$

\section{METODOLOGIA}

\section{Acoplamento da planície de inundação}

Considerou-se uma seção composta por três regiões, como pode ser visto na Figura 3.

O procedimento de cálculo da propagação na planície consiste em utilizar uma tabela com os parâmetros necessários para o modelo na seção. Foi criado um vetor crescente de pro- 
fundidades até um valor arbitrário que fosse suficientemente grande para que a vazão correspondente superasse a vazão máxima nos hidrogramas a montante dos trechos simulados.

Para cada cota, foi calculada uma largura, área, perímetro, raio hidráulico, vazão, celeridade e velocidade (Fórmula de Manning) e parâmetros como o fator beta, o número de Courant e a Difusão, necessários para o modelo Muskingum-Cunge Todini. Para modelos como Muskingum-Cunge não linear, a tabela foi necessária apenas até o cálculo da celeridade.

Durante a aplicação do modelo, foram interpolados (com auxílio da tabela) de acordo com a vazão de referência, os valores necessários para o cálculo dos coeficientes C1, C2 e C3.

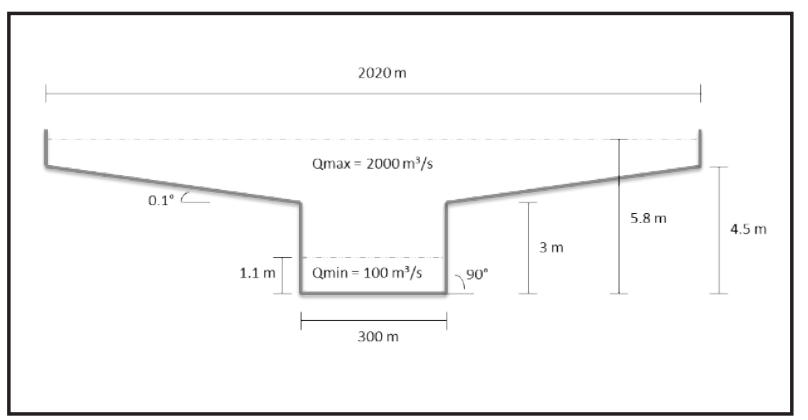

Figura 3 - Planície de inundação utilizada para realizar o exemplo numérico

\section{Comparação entre os modelos MCT, MCNL3 e HEC-RAS}

Os métodos MCT, MCNL3 e HEC-RAS foram aplicados em um canal hipotético composto por três regiões (Figura 3) para avaliar a representatividade da propagação de uma onda de cheia em termos de conservação de volume.

Este experimento numérico consiste em um trecho de canal com seção transversal composta, de largura da base(B), largura da planície (Bp), que corresponde a 2020 m descontados de B, comprimento (L), declividade (S0), coeficiente de Manning da calha (Man), coeficiente de Manning da planície (ManP), discretização espacial $(\Delta \mathrm{x})$ e discretização temporal $(\Delta \mathrm{t})$.Consideramos os seguintes valores dos parâmetros como a simulação de referência: $\mathrm{B}=300 \mathrm{~m} ; \mathrm{Bp}=1720 \mathrm{~m} ; \mathrm{L}=200$ $\mathrm{km} ; \mathrm{S} 0=0,0001 \mathrm{~m} / \mathrm{m} ; \operatorname{Man}=0,035 \mathrm{~m}^{-1 / 3} \mathrm{~s} ; \operatorname{ManP}=0,13 \mathrm{~m}^{-1 / 3} \mathrm{~s}$; $\Delta \mathrm{x}=1000 \mathrm{~m} ; \Delta \mathrm{t}=3600 \mathrm{~s}$.

A Figura 3 mostra a seção transversal para esse teste considerando os parâmetros padrão. A condição inicial do modelo é uma vazão de $100 \mathrm{~m}^{3} / \mathrm{s}$, que corresponde a uma profundidade de 1,1 metros.

Diversas simulações foram realizadas variando os parâmetros S0, Man, $\Delta \mathrm{x}$ e $\Delta$ t. Cada parâmetro foi perturbado, em torno do valor de referência, mantendo os outros parâmetros fixos, de acordo com a Tabela 1.

No presente trabalho é considerado um hidrograma de entrada sintético NERC (1975) apud Todini (2007), na seção de montante, dado por:

$\mathrm{Q}(\mathrm{t})=$ Qbase $+($ Qpico - Qbase $)\left[\frac{\mathrm{t}}{\mathrm{Tp}} \exp \left(1-\frac{\mathrm{t}}{\mathrm{Tp}}\right)\right]^{\beta}$ onde: Qbase $=100 \mathrm{~m}^{3} / \mathrm{s}$; Qpico $=2000 \mathrm{~m}^{3} / \mathrm{s} ; \mathrm{Tp}=120$ horas e $\beta=8$. (Parâmetro de curvatura do hidrograma).

Após as simulações serão comparados e analisados os resultados obtidos por cada modelo de propagação de vazão. $\mathrm{O}$ erro de volume foi calculado pela seguinte fórmula:

$$
\Delta \mathrm{V}(\%)=\frac{\sum \mathrm{I}-\sum \mathrm{Q}}{\sum \mathrm{I}} 100
$$

Tabela 1 - Variação dos parâmetros utilizados na simulação. Os valores em negrito são os parâmetros para a simulação de referência do teste realizado

\begin{tabular}{c|c|c|c|c|c}
\hline S0 & $\mathbf{0 , 0 0 0 1}$ & 0,00025 & 0,0007 & 0,001 & 0,002 \\
\hline Man & 0,01 & 0,02 & $\mathbf{0 , 0 3 5}$ & 0,045 & 0,06 \\
\hline$\Delta \mathrm{x}$ & 500 & $\mathbf{1 0 0 0}$ & 2000 & 4000 & 6000 \\
\hline$\Delta \mathrm{t}$ & 900 & 1800 & $\mathbf{3 6 0 0}$ & 5400 & 7200 \\
\hline
\end{tabular}

\section{Análise dos critérios de aplicabilidade e da escolha do $\Delta x$ ideal}

A análise do $\Delta \mathrm{x}$ ideal foi feita da seguinte forma. Para quatro tipos de declividade $(0,0001 ; 0,00025 ; 0,0007$ e 0,001$)$ foram variados os comprimentos de rio $(50 \mathrm{~km}, 100 \mathrm{~km}$ e $200 \mathrm{~km}$ ) e o número de trechos. O modelo MCT foi testado nessas condições. Em cada caso verificou-se se ocorreram ou não problemas de conservação de volume ou instabilidade nos hidrogramas calculados. Também foram calculados os valores de $\Delta \mathrm{x}$ ideal em cada situação e analisado se esse valor era uma escolha razoável ou se poderia ser adotado outro valor de $\Delta \mathrm{x}$. Essa análise foi feita de acordo com os erros de volume e instabilidades nos hidrogramas.

O procedimento para se analisar os critérios de aplicabilidade definidos por Ponce foi o seguinte: foram calculados os critérios de Onda Cinemática e Difusão e verificado se era possível utilizar os modelos simplificados MCT e MCNL3. Também foi calculado o coeficiente de Nash-Sutcliffe (Nash e Sutcliffe, 1970 apud Perumal e Sashoo, 2007) dos modelos MCT e MCNL3 com relação ao HEC-RAS. Finalmente, foi analisado se os modelos simplificados tiveram um bom coeficiente de Nash-Stucliffe mesmo não estando de acordo com os critérios de aplicabilidade citados nas equações 50 e 51 .

\section{RESULTADOS}

\section{Comparação entre os modelos de escoamento sim- plificados}

Os erros de volume da simulação de referência são apresentados na Tabela 2.

Observa-se que o modelo HEC-RAS apresenta erro de conservação de volume de $0,64 \%$. No entanto, o modelo MCNL3 apresenta um erro elevado de $-19,49 \%$. Finalmente, o modelo MCT apresenta um erro de - $0,06 \%$, o que pode ser considerado desprezível comparado ao erro observado no MCNL3. 
Tabela 2 - Resultados da vazão de pico (Qpico), tempo de pico $(\mathrm{T} p)$ e conservação de volume $(\Delta \mathrm{V} \%)$ na simulação de referência

\begin{tabular}{c|c|c|c}
\hline Modelo & Qpico $\left(\mathrm{m}^{3} / \mathrm{s}\right)$ & $\mathrm{Tp}(\mathrm{h})$ & $\Delta \mathrm{V} \%$ \\
\hline HEC-RAS & 678,29 & 123 & $0,64 \%$ \\
\hline MCT & 656,07 & 121 & $-0,06 \%$ \\
\hline MCNL3 & 526,4 & 94 & $-19,49 \%$ \\
\hline
\end{tabular}

\section{Variação da declividade}

A declividade teve um efeito importante com relação à conservação de volume. A Figura 4 apresenta os erros de volume dos modelos comparados no teste numérico para diferentes declividades. Observa-se que o modelo MCNL3 apresenta erros maiores à medida que $\mathrm{S} 0$ diminui. Os erros são aproximadamente $20 \%$ em valor absoluto no caso da declividade de $0,0001 \mathrm{~m} / \mathrm{m}$.

Os modelos MCT e HEC-RAS apresentam erro de volume insignificante.

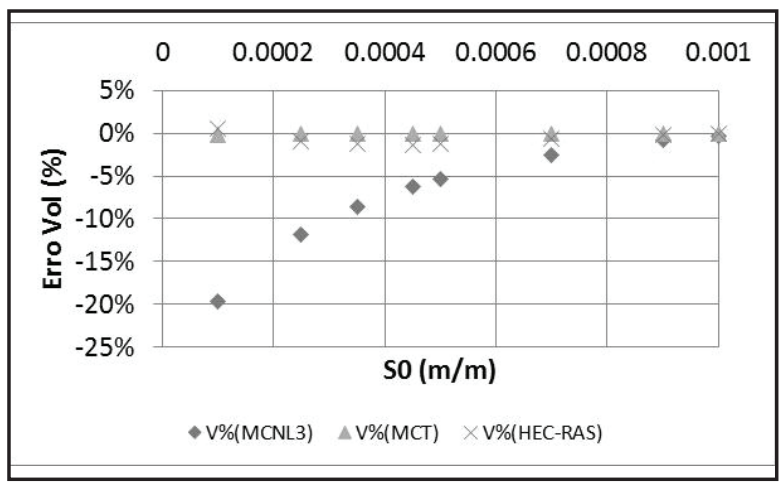

Figura 4 - Erro no volume para diferentes declividades

\section{Variação da rugosidade da calha}

No que diz respeito à rugosidade (Figura 5), MCNL3 apresenta erros de volume crescentes com o aumento do coeficiente de Manning na calha do rio até o valor de $0,06 \mathrm{~m}^{-1 / 3} \mathrm{~s}$. Após esse valor os erros diminuem. Os valores ultrapassam $20 \%$ em valor absoluto para o modelo MCNL3.

Novamente os modelos MCT e HEC-RAS apresentam valores muito baixos que chegam a- 0,34\% (MCT) e - 0,64 (HEC -RAS) na rugosidade $0,06 \mathrm{~m}^{-1 / 3} \mathrm{~s}$ e $0,035 \mathrm{~m}^{-1 / 3}$ s respectivamente.

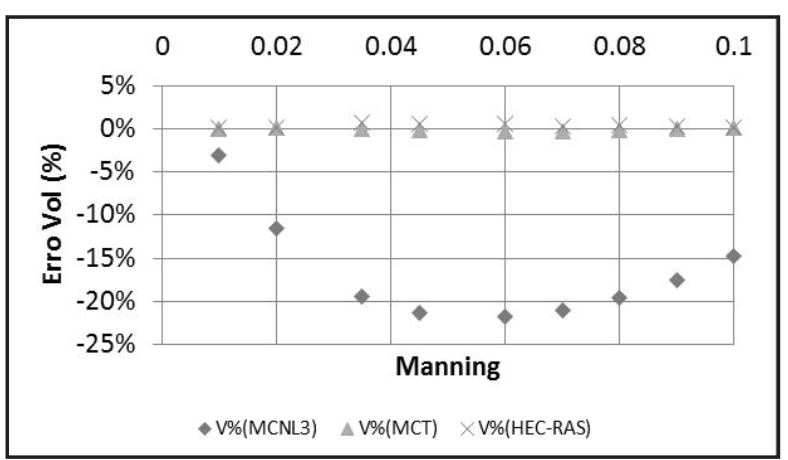

Figura 5 - Erro no volume para diferentes rugosidades do rio

\section{Variação da discretização espacial}

A discretização temporal gerou uma grande diferença nos resultados de erro de volume.

O erro de volume observado na Figura 7 mostra que para o MCT, os erros foram abaixo de 1\% (em módulo) apenas para intervalos espaciais inferiores a 2000 metros. Acima disso, os erros foram mais altos, chegando a $-11,57 \%$ para um $\Delta \mathrm{x}$ de 50000 metros. Para 100 e $200 \mathrm{~km}$ o modelo apresentou instabilidade e subida invertida no gráfico (Figura 6).

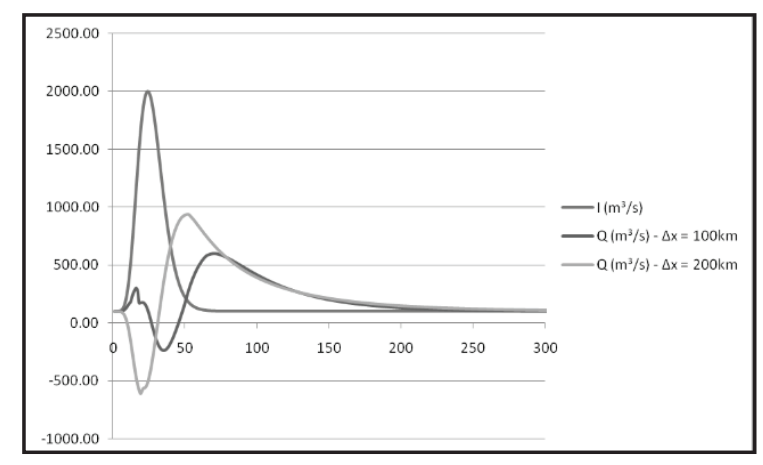

Figura 6 - Problemas de instabilidade numérica e subida invertida do hidrograma. Os hidrogramas são resultados das simulações obtidas pelo modelo MCT

O HEC-RAS apresentou menores erros de volume a partir do valor de $50 \mathrm{~km}$ de intervalo espacial. Em contrapartida, problemas de instabilidade surgiram a partir de um $\Delta \mathrm{x}$ de $20 \mathrm{~km}$.

É importante citar também que a partir de $20 \mathrm{~km} \mathrm{o}$ MCT apresentou vazões negativas na subida do hidrograma simulado (subida invertida). No HEC-RAS esses problemas começaram a aparecer a partir de $5 \mathrm{~km}$.

Os erros de volume do modelo MCNL3 tiveram uma média de $-20,34 \%$. De 50 metros a 5000 metros, de intervalo espacial, o escoamento não chegou a entrar na planície de inundação e a partir de $10 \mathrm{~km}$ observou-se a presença de vazões negativas na subida do hidrograma.

A linha $\Delta \mathrm{x}=6 \mathrm{~km}$ (intervalo espacial ideal) indica qual o limite de escolha de $\Delta x$. Pode-se perceber que para valores menores do que $6 \mathrm{~km}$, os erros do MCT e HEC-RAS são baixos. Após esse valor começam a aparecer problemas na conservação de volume, principalmente para o modelo MCT.

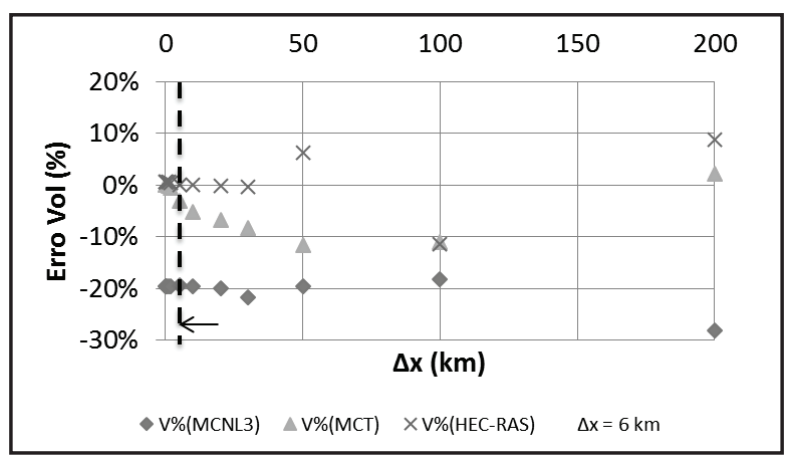

Figura 7 - Erro no volume para diferentes $\Delta \mathrm{x}$ 


\section{Variação da discretização temporal}

Como pode ser visto na Figura 8, o MCT apresentou baixos valores de erro de volume. A média desses valores foi de $-0,10 \%$. O MCNL3 apresentou uma média de $-19 \%$.

Devido ao formato do intervalo temporal de saída do hidrograma calculado pelo HEC-RAS, optou-se em não utilizá-lo nessa análise.

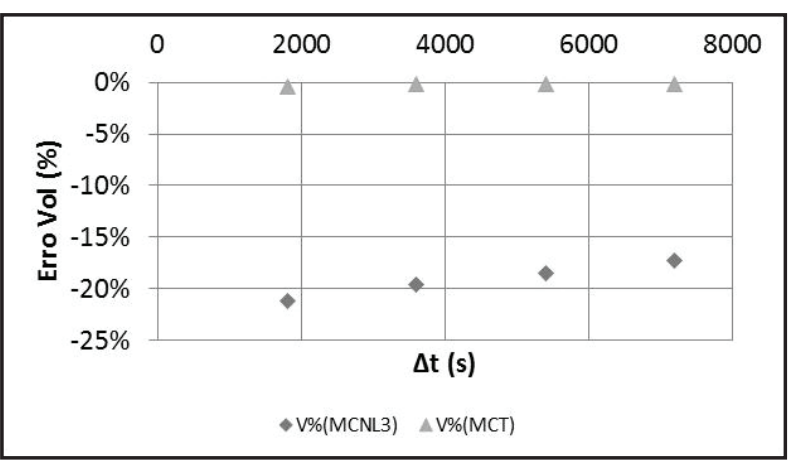

Figura 8 - Erro no volume para diferentes $\Delta t$

\section{Critérios de aplicabilidade}

As últimas análises do exemplo numérico foram relacionadas à escolha do $\Delta \mathrm{x}$ ideal e critérios de aplicabilidade.

Foi calculado um $\Delta \mathrm{x}$ para cada valor de declividade e de comprimento do trecho (Tabela 3). Os campos em vermelho na tabela dizem respeito a comportamentos instáveis nos hidrogramas propagados ou valores de vazões negativas ou abaixo da condição inicial do teste. O número de 1 a 4 em módulo indica que nesse campo apareceram erros de conservação de volume entre $1 \%$ e $4 \%$ em módulo. $\mathrm{O}$ valor $|>4|$, em verde, indica que para essa escolha de $\Delta \mathrm{x}$ e comprimento de rio, os erros de volume superaram 4\% em módulo. Os campos na cor amarela indicam o $\Delta \mathrm{x}$ calculado pela equação 52 citada nesse artigo.

Observando a Tabela 3 percebe-se que para as declividades de $1 \mathrm{~cm} / \mathrm{km}$ e $2.5 \mathrm{~cm} / \mathrm{km}$ o valor de $\Delta \mathrm{x}$ encontrado a partir da equação 52 não foi ideal. Isso devido ao fato de que em 5000 metros (valor de $\Delta x$ ) já existia erros entre 1\% e 4\% em módulo e inconsistências no hidrograma propagado. Para as declividades de $7 \mathrm{~cm} / \mathrm{km}$ e $1 \mathrm{~m} / \mathrm{km}$, o valor de $\Delta x$ calculado pela equação 52 foi menor do que o $\Delta \mathrm{x}$ onde começam os problemas de erro de volume, porém já existiam problemas de vazões negativas ou algum comportamento absurdo no hidrograma propagado. Deve-se destacar que para as declividades menores os problemas de conservação de volume apareceram em menores $\Delta \mathrm{x}$, porém os problemas de vazão negativa na subida do hidrograma simulado surgiram para maiores $\Delta \mathrm{x}$.

Com relação aos critérios de aplicabilidade, propostos por Ponce (1989), pode-se perceber que, apesar de um alto coeficiente de Nash-Sutcliffe (Tabela4), os valores obtidos pelos critérios de aplicabilidade ficaram fora do limite estabelecido por Ponce (1989).

Tabela 3 - Comparação entre o $\Delta x$ definido pela equação 52 e um $\Delta x$ qualquer

\begin{tabular}{|c|c|c|c|c|c|c|c|c|c|c|c|c|c|}
\hline 0.0001 & \multicolumn{12}{|c|}{$\Delta \mathrm{x}(\mathrm{m})$} & 6000 \\
\hline $\mathrm{L}$ & 50 & 100 & 500 & 1000 & 2000 & 5000 & 10000 & 20000 & 30000 & 50000 & 100000 & 200000 & \\
\hline 50000 & & & & & & $|1-4|$ & $|>4|$ & $|>4|$ & $|>4|$ & $|>4|$ & & & \\
\hline 100000 & & & & & & $|1-4|$ & $|>4|$ & $|>4|$ & $|>4|$ & $|>4|$ & $|>4|$ & & \\
\hline 200000 & & & & & & $|1-4|$ & $|>4|$ & $|>4|$ & $|>4|$ & $|>4|$ & $|>4|$ & $|>4|$ & \\
\hline 0.00025 & \multicolumn{12}{|c|}{$\Delta \mathrm{x}(\mathrm{m})$} & 7000 \\
\hline $\mathrm{L}$ & 50 & 100 & 500 & 1000 & 2000 & 5000 & 10000 & 20000 & 30000 & 50000 & 100000 & 200000 & \\
\hline 50000 & & & & & & $|1-4|$ & |1-4| & $|1-4|$ & $|>4|$ & $|>4|$ & & & \\
\hline 100000 & & & & & & $|1-4|$ & $|1-4|$ & $|>4|$ & $|>4|$ & $|>4|$ & $\mid>4$ & & \\
\hline 200000 & & & & & & $|1-4|$ & $|1-4|$ & $|>4|$ & $|>4|$ & $|>4|$ & $|>4|$ & $|>4|$ & \\
\hline 0.0007 & \multicolumn{12}{|c|}{$\Delta \mathrm{x}(\mathrm{m})$} & 9000 \\
\hline $\mathrm{L}$ & 50 & 100 & 500 & 1000 & 2000 & 5000 & 10000 & 20000 & 30000 & 50000 & 100000 & 200000 & \\
\hline \multicolumn{13}{|l|}{50000} & \\
\hline 100000 & & & & & & & & & & $|1-4|$ & $|>4|$ & & \\
\hline 200000 & & & & & & & $|1-4|$ & $|1-4|$ & $|1-4|$ & $|1-4|$ & $1>4$ & $|>4|$ & \\
\hline 0.001 & \multicolumn{12}{|c|}{$\Delta \mathrm{x}(\mathrm{m})$} & 10000 \\
\hline $\mathrm{L}$ & 50 & 100 & 500 & 1000 & 2000 & 5000 & 10000 & 20000 & 30000 & 50000 & 100000 & 200000 & \\
\hline 50000 & & & & & & & & & & $|1-4|$ & & & \\
\hline 100000 & & & & & & & & & & $|1-4|$ & $|1-4|$ & & \\
\hline 200000 & & & & & & & & & $|1-4|$ & $|>4|$ & $|>4|$ & $|>4|$ & \\
\hline
\end{tabular}

Os números nos quadros amarelos correspondem ao $\Delta \mathrm{x}$ ideal conforme a equação 52 para cada declividade. Os valores variando de 50 a 200000 correspondem aos $\Delta \mathrm{x}$ testados. Os valores de 50000 a 200000 correspondem aos comprimentos de trechos testados. Os quadros verdes indicam que não houve problemas de instabilidade ou subida invertida nos hidrogramas. Os quadros vermelhos indica que houve algum desses problemas. Os números, em módulo, indicam os erros de conservação de volume 
Tabela 4 - Comparação dos critérios de aplicabilidade e coeficiente de NS

\begin{tabular}{|c|c|}
\hline \multicolumn{2}{|c|}{ Coeficiente Nash-Sutcliffe (NS) } \\
\hline NS (MCT/HEC-RAS) & $98,81 \%$ \\
\hline NS (MCNL3/HEC-RAS) & $71,78 \%$ \\
\hline \multicolumn{2}{|c|}{ Critérios de aplicabilidade - Ponce } \\
\hline Onda cinemática & 2,02 \\
\hline Difusão & 15,62 \\
\hline
\end{tabular}

A Figura 9 mostra a partir de qual valor de declividade poderia ser utilizado os modelos de onda cinemática (semelhantes ao MCT e MCNL3) em um gráfico de erro de volume e variação de declividade. Observa-se que apenas a partir de 4 $\mathrm{m} / \mathrm{km}$, que corresponde ao critério de aplicabilidade de modelos tipo onda cinemática, os modelos MCT e MCNL3 poderiam ser utilizados. Observa-se que o modelo MCNL3 tem erros de volume consideráveis quando aplicado em declividades inferiores. Esse problema não é observado no MCT, que apesar de ser um modelo simplificado tem características de conservação de volume semelhante ao HEC-RAS.

\begin{tabular}{|c|c|c|c|c|c|}
\hline \multirow{11}{*}{ 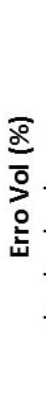 } & \multirow{9}{*}{$\begin{array}{r}5 \% \\
0 \% \\
-5 \% \\
-10 \% \\
-15 \% \\
-20 \%\end{array}$} & \multicolumn{2}{|c|}{0.002} & 0.004 & 0.00 \\
\hline & & & & $\mathrm{T}$ & \\
\hline & & 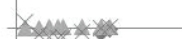 & & 1 & \\
\hline & & & & 1 & \\
\hline & & - & & i & \\
\hline & & $\bullet$ & & 1 & \\
\hline & & & & 1 & \\
\hline & & 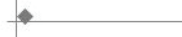 & & KW & \\
\hline & & & & $\longrightarrow$ & \\
\hline & & & $\mathrm{SO}(\mathrm{m}$ & /m) & \\
\hline & & $\bullet$ V\%(MCNL3) & $\triangle \mathrm{V} \%(\mathrm{MCT})$ & $\times$ V\%(HEC-RAS) & \\
\hline
\end{tabular}

Figura 9 - Erros de volume dos modelos testados para diferentes declividades. A linha vertical mostra o limite de aplicabilidade de onda cinemática proposto por Ponce (1989). A seta indica que modelos do tipo onda cinemática deveriam ser utilizados quando a declividade do rio fosse maior do que $0,004 \mathrm{~m} / \mathrm{m}$

\section{DISCUSSÕES E CONCLUSÃO}

A metodologia proposta por Todini (2007), aqui chamada de MCT, mostrou-se satisfatória no exemplo numérico.

Em geral, o modelo MCNL3 foi pior do que o modelo MCT e o HEC-RAS. Isso é esperado e foi observado em outros trabalhos (FAILLACHE et al., 2004; TANG; SAMUELS, 1999; PONTES; COLLISCHONN, 2012).

Quando se variou a declividade, os modelos MCT e HEC-RAS conservaram o volume. Os erros de volume foram muito pequenos, inferiores a $1,2 \%$.

O MCNL3 gerou erros de volume na ordem de $-20 \%$ para declividades mais baixas. Os erros de volume nessas declividades eram esperados, pois são nessas declividades que o efeito do termo de pressão da equação dinâmica começa a fazer diferença (TODINI, 2007; CAPPELAERE, 1997).
É importante citar que os erros de volume são maiores nas declividades mais baixas, porém é nos rios mais íngremes que acontecem os problemas de instabilidade.

A variação da rugosidade não influenciou muito no erro de volume dos modelos MCT e HEC-RAS. Os erros observados podem ser considerados desprezíveis perto dos erros do modelo MCNL3.

O modelo MCT apresenta certa sensibilidade aos valores de $\Delta \mathrm{x}$ adotados na discretização espacial. Valores muito altos de $\Delta \mathrm{x}$ podem originar erros de vazão de pico e tempo de ocorrência do pico, além de problemas nos hidrogramas calculados, como a "subida invertida" ou instabilidade. Os mesmos problemas podem ser identificados no modelo HEC-RAS quando adotado $\Delta \mathrm{x}$ altos. A partir de 5000 metros o MCT já apresentava alguns erros de volume. O HEC-RAS só apresentou erros de volume a partir de $50 \mathrm{~km}$ de intervalo espacial. Em contrapartida, a instabilidade no hidrograma foi percebida em um $\Delta \mathrm{x}$ de $20 \mathrm{~km}$.

A adoção do valor ideal de $\Delta \mathrm{x}$ indicado pela equação 52 não garante que alguns destes problemas não vão ocorrer. Os resultados parecem indicar que o $\Delta \mathrm{x}$ ideal deve ser menor do que o valor indicado pela equação 52 . Não parece haver problemas em adotar valores de $\Delta \mathrm{x}$ relativamente pequenos, embora o tempo de processamento possa ser um pouco maior. Tomando por base os resultados poderia se propor a utilização de um $\Delta \mathrm{x}$ ideal até três vezes menor do que o $\Delta \mathrm{x}$ ideal sugerido pela equação 52 .

Com relação à escolha do $\Delta \mathrm{x}$ ideal pode-se perceber que em alguns testes os erros de volume, por exemplo, aparecem até antes do $\Delta \mathrm{x}$ ideal. Analisando os resultados pode-se concluir que o $\Delta \mathrm{x}$ mostrado na equação 52 não deve ser considerado como ideal. É preferível o uso de discretizações espaciais sempre menores que o valor supostamente ideal.

É possível também estabelecer um novo valor ideal com base na mesma formulação. Com base nos resultados, este trabalho sugere que seja utilizado um valor para intervalo ou discretização espacial de:

$$
\mathrm{DX} \text { ideal }=\mathrm{DX} / 3 \text {, }
$$

onde Dx é calculado pela equação 52. Esse novo valor irá diminuir a ocorrência de erros de volume, subida invertida e instabilidade nos hidrogramas.

Deve-se frisar que esse valor é uma sugestão e não está validado, precisando assim de vários outros testes variando hidrogramas de entrada, seções transversais e parâmetros para que seja validado.

Com relação ao intervalo temporal utilizado, o MCT não apresentou erros de volume significativos com a variação desse parâmetro.

Acerca da análise dos critérios de aplicabilidade estabelecidos por Ponce (1989), os resultados sugerem que o modelo MCT pode ser utilizado fora dos limites de aplicabilidade de modelos tipo Onda Cinemática. Apesar de não poder ser utilizado, de acordo com o valor do critério de Onda Cinemática, o modelo MCT apresentou um coeficiente NS de 98,81\% com relação ao HEC-RAS. Analisando apenas esse coeficiente podese concluir que o modelo MCNL3 também pode ser utilizado 
fora dos critérios de aplicabilidade.

Uma análise mais aprofundada foi feita observando os erros de volume para diferentes declividades e o valor de aplicabilidade de modelos tipo Onda Cinemática. Os resultados mostram que modelos tipo onda cinemática (semelhantes ao MCT e MCNL3) só poderiam ser utilizados em declividades superiores a $4 \mathrm{~m} / \mathrm{km}$. O erro de volume mostra que o modelo MCNL3 apresenta muitos erros em declividades menores que $4 \mathrm{~m} / \mathrm{km}$, porém o modelo MCT apresentou erros praticamente nulos. Aparentemente percebe-se que o modelo MCT pode ser utilizado mesmo que os critérios de aplicabilidade não sejam respeitados.

Com relação ao equacionamento da planície de inundação e o seu acoplamento nos modelos simplificados, pode-se concluir que o equacionamento da planície é simplificado, mas representa bem a propagação de vazão na planície. Essa afirmação pode ser considerada devido a semelhança dos resultados dos modelos MCT e HEC-RAS com planície de inundação.

A implementação do equacionamento de planície de inundação nos modelos simplificados, é de grande importância devido à quantidade de rios que apresentam essa característica e de modelos hidrológicos que utilizam métodos simplificados para propagação da vazão no rio.

O modelo MCT com planície de inundação teve os resultados tão bons quanto o modelo hidrodinâmico HEC-RAS.

Em síntese, o MCT com planície de inundação permite aliar os benefícios de modelos não lineares, a fácil implementação, a conservação de volume ou a diminuição significativa dos erros. Sendo assim pode-se concluir que o MCT pode ser utilizado para substituir um modelo hidrodinâmico em muitas aplicações, desde que não existam efeitos de jusante, os quais o modelo MCT não pode representar.

\section{Limitação dos modelos}

Uma atenção especial foi dada aos erros que podem ser observados diretamente no gráfico do hidrograma, como a tendência de alguns modelos apresentarem uma pequena redução da vazão antes do início da ascenção do hidrograma.

Este efeito, chamado aqui de subida invertida do hidrograma, foi observado por outros autores (PERUMAL, 1992; SZÉL; GÁSPÁR, 2000) e está relacionado à discretização temporal e espacial adotada. Além disso, a avaliação visual também

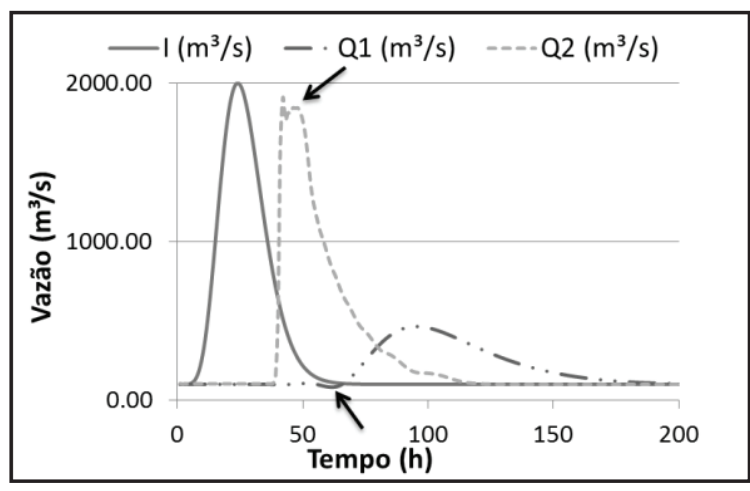

Figura 10 - Erros de instabilidade (hidrograma Q2) e erros de subida invertida do hidrograma (hidrograma Q1) buscou identificar períodos de instabilidade nos picos e na recessão, como ilustrado no exemplo da Figura 10.

\section{AGRADECIMENTOS}

Ao CNPq pela concessão de bolsa de mestrado ao primeiro autor no período de 2009 à 2011 .

\section{REFERÊNCIAS}

CAPPELAERE, B. Accurate Diffusive Wave Routing. J. Hydraulic Eng., ASCE, v. 123, n.3, p. 174-181, 1997.

CUNGE, J.A.; HOLLY, F.M.; VERNEY, A. Practical aspects of computacional river bydraulics. London: Pitman Advanced. XVI, 1980. 420p.

FAILACHE, N. G.; ALLASIA, D.; COLLISCHONN, W. Avaliação do Método de Muskingum-Cunge não linear com conservação de volume para propagação de cheias em rios. In: SIMPÓSIO BRASILEIRO DE RECURSOS HÍDRICOS, XIX., 2004, Maceió: ABRH, 2004.

HORRITT, M. S.; BATES, P. D. (2002). Evaluation of 1D and 2D numerical models for predicting river flood inundation. Journal of Hydrology, v. 268, p. 87 - 99, 2002.

JONES, S. B. Choice of space and time steps in the MuskingumCunge flood routing method. Proceedings - Institution of Civil Engineers, part 2. 71, p. 759 - 772, 1981.

KOUSSIS, A.D. Assessment and review of the hydraulics of storage flood routing 70 years after the presentation of the Muskingum method. Journal Hydrological Sciences, v.54, n.1, p. 43-61, 2009.

NASH, J.E.; SUTCLIFFE, J. V. (1970). River flow forecasting through conceptual models, Part 1: A discussion of principles. Journal of Hydrology, v. 10, n. 3, p. $282-290,1970$.

PAIVA, R. C. D. Modelagem Hidrológica e Hidrodinâmica de Grandes Bacias. Estudo de caso: Bacia do rio Solimões. 2009. Dissertação (Mestrado) - Universidade Federal do Rio Grande do Sul, IPH, 2009.

PAZ, A. R.; COLLISCHONN, W.; TUCCI, C.E.M. Simulação hidrodinâmica integrada rio-planície em ambiente SIG: aplicação ao Rio Aquidauana. SIMPÓSIO DE GEOTECNOLOGIAS NO PANTANAL, I ${ }^{\circ}$ - GEOPANTANAL. Campo Grande MS, (2006).

PAZ, A. R. Simulação Hidrológica de Rios com Grandes Planícies de Inundação. 2010. Tese (Doutorado) - Universidade Federal do Rio Grande do Sul, IPH, 2010. 
PERUMAL, M. The cause of negative initial outflow with the Muskingum method / Cause d'un débit de sortie négatifobtenupar la méthode de Muskingum, Hydrological Sciences Journal, v. 37, n. 4, p. 391-401, 1992.

PERUMAL, M. Hydrodynamic derivation of a variable parameter Muskingum method. Theory and solution procedure. Hydrological Sciences, v. 39, n.1, 1994.

PERUMAL, M. .Hydrodynamic derivation of a variable parameter Muskingum method. Verification. Hydrological Sciences, v. 39, n. 2, 1994

PERUMAL, M.; O'CONNELL, P.E.O.; RANGA RAJU, K. G. (2001).Field applications of a variable-parameter Muskingum Method. Journal of Hydrologic Engineering, v. 6, n. 3, p. 196-206, 2001.

PERUMAL, M.; SAHOO, M. Applicability criteria of the variable parameter Muskingum stage and discharge routing methods. Water Resources Research, v.43, 2007.

PONCE, V. M. Engineering Hydrology: principles and practices. Prentice Hall., 1989. 640p.

PONTES, P. R. M.; COLLISCHONN, W. Conservação de Volume em Modelos Simplificados de Propagação de Vazão. Revista Brasileira de Recursos Hidricos, v. 17, p. 83-96, 2012.

PRICE, R. K. Volume-Conservative Nonlinear Flood Routing. Journal of Hydraulic Engineering, v. 135, n. 10, p. 838-845, 2009.

SILVA, R. C. V.; MASCARENHAS, F. C. B.; MIGUEZ, M. G. Hidráulica Fluvial. Rio de Janeiro: COPPE/ UFRJ, 2003.

SZÉL, S.; GÁSPÁR, C. On the negative weighting factors in the Muskingum-Cunge scheme. Journal of Hydraulic Research, v. 38, n. 4, p. 299-306, 2000.

TANG, X.; SAMUELS, P. G. Variable Parameter MuskingumCunge Method for flood routing in a compound channel. Journal of Hydraulic Research., v. 37, p. 591-614, 1999.

THOMPSON, J.R.; SORENSON, H.R.; GAVIN, H.; REFSGAARD, A. Application of the coupled MIKE SHE/ MIKE SHE 11 modeling system to a lowland wet grassland in southeast England. Journal of Hydrology, 2004.

TODINI, E. A mass conservative and water storage consistent variable parameter Muskingum-Cunge approach. Hydrologyand Earth System Sciences, v. 4, p. 1549-1592, 2007.

TUCCI, C. E. M. Modelos Hidrológicos. 2.ed. Porto Alegre: Editora da UFRGS, 2005.

USACE. HEC-RAS River Analysis System. Release Notes. US Army Corps of Engineers. Hydrologic Engineering Center.Version 4.0.0., 2008.
ZHANG, Z.; WANG, S.; SUN, G.; MCNULTY, S. G.; ZHANG, H.; LI, J.; ZHANG, M.; KLAGHOFER, E.; STRAUSS, P. Evaluation of the mike she model for application in the loess Plateau, China. Journal of the American Water Resources Association, 2008. 